\title{
Current forms of inhibitory training produce no greater reduction in drinking than simple assessment
}

Citation for published version (APA):

Smith, J. L., Dash, N. J., Johnstone, S. J., Houben, K., \& Field, M. (2017). Current forms of inhibitory training produce no greater reduction in drinking than simple assessment: A preliminary study. Drug and Alcohol Dependence, 173, 47-58. https://doi.org/10.1016/j.drugalcdep.2016.12.018

Document status and date:

Published: 01/04/2017

DOI:

10.1016/j.drugalcdep.2016.12.018

Document Version:

Publisher's PDF, also known as Version of record

Document license:

Taverne

Please check the document version of this publication:

- A submitted manuscript is the version of the article upon submission and before peer-review. There can be important differences between the submitted version and the official published version of record.

People interested in the research are advised to contact the author for the final version of the publication, or visit the DOI to the publisher's website.

- The final author version and the galley proof are versions of the publication after peer review.

- The final published version features the final layout of the paper including the volume, issue and page numbers.

Link to publication

\footnotetext{
General rights rights.

- You may freely distribute the URL identifying the publication in the public portal. please follow below link for the End User Agreement:

www.umlib.nl/taverne-license

Take down policy

If you believe that this document breaches copyright please contact us at:

repository@maastrichtuniversity.nl

providing details and we will investigate your claim.
}

Copyright and moral rights for the publications made accessible in the public portal are retained by the authors and/or other copyright owners and it is a condition of accessing publications that users recognise and abide by the legal requirements associated with these

- Users may download and print one copy of any publication from the public portal for the purpose of private study or research.

- You may not further distribute the material or use it for any profit-making activity or commercial gain

If the publication is distributed under the terms of Article $25 \mathrm{fa}$ of the Dutch Copyright Act, indicated by the "Taverne" license above, 
Full length article

\title{
Current forms of inhibitory training produce no greater reduction in drinking than simple assessment: A preliminary study
}

\author{
Janette L. Smith ${ }^{\mathrm{a}, *}$, Nicole J. Dash ${ }^{\mathrm{b}}$, Stuart J. Johnstone ${ }^{\mathrm{b}}$, Katrijn Houben ${ }^{\mathrm{c}}$, Matt Field ${ }^{\mathrm{d}, \mathrm{e}}$ \\ a National Drug and Alcohol Research Centre, University of New South Wales, Sydney, NSW 2052, Australia \\ b School of Psychology, University of Wollongong, Wollongong, Australia \\ c Clinical Psychological Science, Maastricht University, Maastricht, The Netherlands \\ d Department of Psychological Sciences, University of Liverpool, Liverpool, United Kingdom \\ e UK Centre for Tobacco and Alcohol Studies, Liverpool, United Kingdom
}

\section{A R T I C L E I N F O}

\section{Article history:}

Received 18 October 2016

Received in revised form 6 December 2016

Accepted 14 December 2016

Available online 31 January 2017

\section{Keywords:}

Alcohol

Inhibitory control training

Go/NoGo

Stop-Signal

Assessment

Hawthorne effect

\begin{abstract}
A B S T R A C T
Background: Disinhibition is apparent in users of many substances, including heavy drinkers. Previous research has shown that brief training to improve inhibitory control is associated with reduced alcohol consumption. We investigated whether a new form of inhibitory training would produce greater reductions, relative to a carefully designed control condition and a proven method of reducing consumption, the Brief Alcohol Intervention (BAI).

Methods: One hundred and fourteen regular drinkers were assigned randomly to one of five training conditions: Control (no inhibitory training); Beer-NoGo (inhibit responses linked to task-irrelevant pictures of beer); Restrained-Stop (requiring more urgent inhibition but without pictures of beer); Combined (a previously untested form of training requiring urgent inhibition to pictures of beer); or BAI. The outcome measures were alcohol consumption in the week before and after training, and in a bogus taste test administered immediately post-training.

Results: Participation in the study, regardless of condition, was associated with reductions in weekly consumption. However, only the BAI produced a greater reduction relative to the Control condition. The training tasks were not associated with reductions in taste test consumption.

Conclusions: Although concerns about low power limit confidence, the current study suggests that three forms of inhibitory training do not have a substantial effect on drinking beyond the effect of simple assessment, in comparison to a control task which does not promote impulsive responding. Future research needs to establish a training protocol that produces greater reductions in consumption not only relative to the effect of assessment but also relative to a BAI.
\end{abstract}

(c) 2017 Elsevier B.V. All rights reserved.

\section{Introduction}

Inhibition is the ability to withhold, stop, or delay an inappropriate response (Barkley, 1997; Diamond, 2013); the cessation of an immediate response allows time for other important psychological processes to evaluate the situation, and select and execute a more appropriate response (Barkley, 1997). Deficits in inhibitory control feature prominently in new models of the development, maintenance, and relapse of substance use disorders (e.g., Hester et al., 2010; Jentsch and Pennington, 2014), and failures of control are implicated in DSM-5 criteria involving using a substance more, or more often, than intended, and consistently failed efforts to limit use (American Psychiatric Association, 2013). Inhibition

\footnotetext{
* Corresponding author.

E-mail address: janette.smith@unsw.edu.au (J.L. Smith).
}

deficits have been confirmed experimentally in users of a range of substances including not only alcohol dependence, but also heavy drinkers (reviewed in Smith et al., 2014).

If it is accepted that an inhibitory deficit is associated with undesirable and/or risky behaviours in these disorders, then the corollary is that training to improve this deficit may decrease these behaviours. Several studies have examined whether alcohol consumption in social drinkers can be reduced with training on an inhibitory task such as the Go/NoGo or Stop-Signal task. In several studies using a modified version of the Go/NoGo task (Bowley et al., 2013; Houben et al., 2011, 2012), the letters P and F, each $50 \%$, were superimposed on images of beer and water. For half the participants, the beer image was paired with the Go stimulus (requiring a fast button press response, "Beer-Go" condition), while for the other half, the beer image was paired with the NoGo stimulus (requiring the response to be withheld, "Beer-NoGo" condition). Although the images were irrelevant to the task (i.e., the 
instructions focused on making or withholding responses to the letter stimuli), consistent pairing of the beer image with response inhibition should increase inhibitory control over beer stimuli (that is, should train direct associations between alcohol cues and stopping; Best et al., 2016; Bowditch et al., 2016). Indeed, participants in the Beer-NoGo condition decreased their consumption of alcohol in the week after compared to the week before inhibitory training (Houben et al., 2011, 2012; but see Bowley et al., 2013 for no effect). Some studies additionally use a bogus taste test to measure immediate alcohol consumption (for a review see Jones et al., 2016a). Here, participants are presented with a known amount of alcohol and asked to consume as much or as little as desired in order to rate the drink on several dimensions. The participant is not aware that the experimenter will later measure the amount of alcohol consumed. Training on the Beer-NoGo task is associated with a trend to reduced alcohol consumption in the taste test (Bowley et al., 2013; Houben et al., 2011). Inhibitory control training has also been studied in relation to other health behaviours such as food choices; across domains, the effect size for Go/NoGo tasks has been confirmed to be medium-sized and robust by two recent independently conducted meta-analytic reviews (Allom et al., 2016: 0.50; Jones et al., 2016b: 0.47).

The Stop-Signal task can also be used to assess inhibition; in this task, fast choice responses are required to two primary stimuli (e.g., respond with the left or right hand) and the occasional presentation of a stop-signal indicates the participant should interrupt the button press response (Logan and Cowan, 1984; Logan et al., 1984). In studies linking performance of a Stop-Signal task with subsequent alcohol consumption (Jones et al., 2011a,b), participants were instructed to be especially restrained (i.e., successful inhibition was emphasized over fast responding) or disinhibited (i.e., fast responding was emphasized over successful inhibition). After training, participants in the restrained condition consumed less alcohol in the bogus taste test (Jones et al., 2011a,b), but those studies did not examine changes in weekly alcohol consumption. A different variation on the Stop-Signal task was tested by Jones and Field (2013), in which alcohol-related or neutral pictures served as the Go stimuli, and, for different conditions, $90 \%$ of stop-signals occurred on alcohol trials (alcohol restraint condition) or on neutral picture trials (neutral restraint condition). A third group were instructed to ignore the stop-signal and respond to all pictures (disinhibited condition). In the bogus taste test, participants in the alcohol restraint condition drank less beer than the neutral and disinhibited conditions, which did not differ; weekly consumption was unaffected. Thus, inhibitory training appears to alter alcohol consumption measured both via an immediate taste test, and in standard drinks per week before and after the experimental session (although not all studies report reductions in weekly consumption: Bartsch et al., 2016). Recent meta-analyses have estimated the effect size for Stop-Signal tasks to be robust, albeit smaller than that for Go/NoGo tasks (Allom et al., 2016: 0.26; Jones et al., 2016b: 0.23). Two explanations are possible for the smaller effect: one is that, at least for the early versions of inhibitory training using the StopSignal task (Jones et al., 2011a,b), the task does not associatively link alcohol with inhibition, despite alteration of associations being a principle of cognitive bias modification (MacLeod and Grafton, 2016). Secondly, Jones et al. (2016b) argued that the smaller effect in the Stop-Signal task is due to the fact that these tasks typically involve about $50 \%$ failed inhibitions, and that appetitive cues need to be reliably paired with successful inhibition in order for inhibitory training to reduce alcohol consumption (Jones et al., 2016b).

In the current study, we improve upon the previous research in three respects. The first relates to the control conditions to which the inhibitory training conditions are compared, and how the selection of the control condition may alter the results observed. For the Beer-NoGo task, consumption is often compared to the Beer-Go task, in which alcohol is paired with response execution (Bowley et al., 2013; Houben et al., 2011, 2012). It could be argued that such pairing of alcohol with fast responses may lead to impulsive responding and therefore greater alcohol consumption in the taste test. Thus, for the taste test, it is not clear whether differences between Beer-NoGo and Beer-Go conditions represent low consumption in the Beer-NoGo condition, or high consumption in the Beer-Go condition (or both). Indeed, the interaction between time and condition for weekly consumption is at least partly due to an increase in consumption for the Beer-Go condition in Houben et al. (2011), and a similar, although not significant, pattern was observed in Houben et al. (2012). Similarly, for Jones et al. (2011b), performance in the Restrained condition was compared with the Disinhibited condition, and thus it is difficult to interpret taste test differences between the conditions in that study also. In a subsequent study (Jones et al., 2011a), a Control condition was included which received the usual Stop-Signal task instructions (to balance speed and accuracy); results indicated that participants in the Restrained condition consumed less beer in the taste test than the Control and Disinhibited conditions, which did not differ. This is the clearest evidence of inhibitory training producing a reduction in alcohol consumption, yet the Stop-Signal task even with standard instructions still requires (and therefore trains) inhibition. In the current study we include a Control condition, involving a task with similar stimuli requiring attention, discrimination, and a motor response; however, Go stimuli are $25 \%$ of trials in the Control task. This means that the prepotent response (on NoGo trials, $75 \%$ ) is to do nothing, and only activate a response occasionally. Thus, the Control task here cannot be said to require inhibition, but nor would it favour impulsive responding; furthermore, the task uses neutral (non-alcohol-related) stimuli. However, more importantly, our Control condition allows us to examine the effect of assessment alone on alcohol consumption. Known as subject reactivity, or the Hawthorne effect, changes in a behavior simply due to observation of that behavior were first described in 1933 (Mayo, 1933); demonstrations of reductions in alcohol consumption due to assessment have been noted since 1974 (Bartsch et al., 2016; Gallen, 1974; Kypri et al., 2007; McCambridge and Day, 2008; McCambridge and Kypri, 2011; see Clifford and Maisto, 2000, for a review). Not only have the Beer-NoGo and Restrained-Stop conditions been improperly compared to conditions which increase drinking, but the treatment effect of these interventions has so far been confounded with the assessment effect. Participants in the Control condition are expected to reduce their drinking in the week after compared to the week before taking part in the experiment (due to an effect of assessment); participants in conditions which perform an inhibitory training task must therefore decrease their consumption significantly more than those in the Control condition, in order for the inhibitory task to be considered an effective intervention.

Secondly, we also consider the effectiveness of inhibitory training relative to an established method of reducing consumption, namely a Brief Alcohol Intervention (BAI), which consists of questions about and motivational feedback concerning alcohol consumption. Meta-analytic reviews confirm BAIs are effective at reducing consumption (e.g., Bertholet et al., 2005); they are also effective within the specific target population of this study (i.e., university students; Kypri et al., 2009; Samson and Tanner-Smith, 2015). BAIs can easily reach large samples via the internet; although the inhibitory tasks above could theoretically be delivered online (Jones et al., 2014), in the studies cited above, participants have completed the sessions in the laboratory. In order to justify the extra time and effort associated with laboratory testing, inhibitory tasks should also be at least as effective as a BAI at reducing alcohol consumption. Indeed, Bowley et al. (2013) report that the BAI and Beer-NoGo conditions did not differ at taste test; participants 
in those conditions both consumed significantly less alcohol than those in the Beer-Go condition. In the current study, we include not only a carefully selected Control task but also a BAI condition.

Thirdly, we improve on previous research by more strongly linking alcohol with inhibition in a new version of inhibitory task. Evidence suggests that inhibitory deficits in heavy drinkers are greater when alcohol-related compared to neutral stimuli are used (e.g., Noël et al., 2007; Weafer and Fillmore, 2012), however, the studies using the basic version of the Stop-Signal task (Jones et al., 2011a, 2011b) do not attempt to link alcohol with inhibition, with participants discriminating between and responding to neutral letters $\mathrm{X}$ and $\mathrm{O}$. The modified version of the Stop-Signal task (Jones and Field, 2013) has a stronger link between alcohol and inhibition, with $90 \%$ of inhibition trials occurring in the context of an alcohol image (for the alcohol restraint condition). However, in all three studies, the stop-signal indicating that inhibition is required is not alcohol-related, but rather, a neutral auditory tone. Similarly, studies using Beer-NoGo tasks have a consistent link between alcohol-related images and response inhibition (Bowley et al., 2013; Houben et al., 2011, 2012); however, since participants are instructed to respond according to the superimposed letter stimuli, it could be argued that they do not necessarily process the alcohol and water images. Best et al. (2016) have indeed recently argued that learning of stimulus-stop associations is most effective when images are directly relevant to the task. In the current study we test a new modified Stop-Signal task that directly links alcohol to the need for inhibition. In our new task, participants view images of water with the letters $P$ and $F$ superimposed, and respond with the left and right hand to those letters. The stopsignal was a change in the background image from water to beer. In this way, we combine the urgent need for inhibition afforded by the Stop-Signal task with alcohol-related images, and alcohol itself is the cue for inhibition.

Thus, in the current study we examine alcohol consumption associated with training on one of three inhibitory tasks: not only the Restrained-Stop and Beer-NoGo tasks as investigated previously (Bowley et al., 2013; Houben et al., 2011, 2012; Jones et al., 2011a,b), but also a new Combined task where alcohol itself is the cue for inhibition. We also include a BAI condition as well as a carefully designed Control condition to test the efficacy of inhibitory training against the gold-standard and against nonspecific means of reducing alcohol consumption. Participants were randomly assigned to one of these five conditions and completed two sessions one week apart in the laboratory; they were assessed on alcohol consumption in the laboratory (in a taste test administered after completing the inhibitory training, control task, or BAI), and outside the laboratory (consumption in the week before compared to after completion of the inhibitory training, control task, or BAI). For weekly consumption, we expected that alcohol consumption would reduce between sessions due to non-specific effects (i.e., a main effect of time), and additionally we expected that inhibitory training and the BAI would produce significantly greater reductions in consumption compared to the Control condition (i.e., significant time by condition interactions), with the largest reductions observed in the new Combined task. For taste test consumption, we expected that inhibitory training and the BAI would be associated with reduced alcohol consumption relative to Controls (i.e., condition main effects).

\section{Methods}

\subsection{Participants}

Participants were 114 adults recruited via advertisements on campus and from online research participation websites at the Uni- versity of New South Wales and University of Wollongong (site of testing was included as a factor in preliminary analyses and not found to significantly alter the results). Participants were eligible to participate if they were aged 18-30, liked beer, consumed at least 4 standard drinks in the week prior to testing (1 Australian standard drink $=10 \mathrm{~g}$ alcohol), reported that they were not pregnant, and had no other contraindications to consuming alcohol (e.g., medical conditions, drug interactions). The recruitment flyers, website, initial information sheet and consent form stated that the study investigated the relationship between attitudes and preferences for beer, and reaction time on computer tasks. Participants completed one session of $60 \mathrm{~min}$ and a second session of $30 \mathrm{~min}$ one week later, and were reimbursed $\$ 25$ for their time at the end of the second session, or received points towards partial course requirements for first year psychology courses. The experimental protocol was approved by the University of New South Wales Human Research Ethics Committee before data collection began.

\subsection{Conditions and tasks}

Participants were assigned pseudo-randomly to one of five conditions (Control, Beer-NoGo, Restrained-Stop, Combined, or BAI), indicating which intervention/training task they would complete. We aimed throughout to balance the age, gender, and time of the day tested between conditions, since it was expected (and confirmed, see Results) that participants would consume less in the taste test if they completed the session in the morning. Participants were not aware that they had been assigned to a particular condition until debriefing. Computerised tasks were delivered via Presentation software.

2.2.1. Control condition. Participants assigned to the Control condition completed an oddball task, requiring attention, stimulus discrimination, and a motor response, but not requiring behavioural inhibition. White capital letters $F$ and $P$ were displayed for $800 \mathrm{~ms}$ in the centre of a black screen, with stimulus onset asynchrony (SOA) of 1500 ms mean (range 1200-1800 ms). Participants were instructed to respond to one letter (the designated Go letter, counterbalanced between participants) by pressing the button ('space bar' on a standard QWERTY keyboard), and not to respond to the other letter (the designated NoGo letter). One-quarter of trials were Go trials. Note that in this context NoGo trials are associated with a 'do nothing' response representation, rather than requiring active inhibition; support for this notion comes from event-related potential studies showing a lack of inhibitory increases in the N2/P3 complex when the NoGo stimulus is frequent (e.g., Nieuwenhuis et al., 2003) or otherwise expected (e.g., Randall and Smith, 2011). Participants completed a practice block of 10 trials ( $3 \mathrm{Go}$ ) with one index finger, then 2 experimental blocks of 64 trials each with the same hand, then were asked to use the other index finger for another practice block of 10 trials and 2 experimental blocks of 64 trials. The starting hand was also counterbalanced between participants. If participants did not respond within $800 \mathrm{~ms}$ on Go trials, the Go stimulus was replaced with the words "TOO SLOW" for $500 \mathrm{~ms}$, and then a black screen until the next trial began.

2.2.2. Beer-NoGo condition. In the Beer-NoGo task, white capital letters $\mathrm{F}$ and $\mathrm{P}$ were displayed for $800 \mathrm{~ms}$ centrally on top of images of water or beer, with a black background, and with SOA of 1500 ms mean (range 1200-1800 ms). Participants were instructed to respond to one letter (the designated Go letter, counterbalanced between participants) by pressing the button (space bar), and not to respond to the other letter (the designated NoGo letter). Three-quarters of trials were Go trials; although this is different to previous research using the Beer-NoGo task (Bowley et al., 2013; Houben et al., 2011, 2012), and halves the number of instances 
on which alcohol is paired with inhibition, we wanted to ensure equal inhibition probability with the Restrained-Stop and Combined tasks at $25 \%$, and that inhibition would be difficult since it is rarely required. Although the images were irrelevant to the task, images of beer were always displayed behind the NoGo letter, and images of water were always displayed behind the Go letter. The same four images of each were used as in previous studies (Houben et al., 2011, 2012; Bowley et al., 2013). Participants completed a practice block of 10 trials ( $7 \mathrm{Go}$ ) with the index finger of one hand, then 2 experimental blocks of 64 trials each with the same hand, then were asked to use the other index finger for another practice block of 10 trials and 2 experimental blocks of 64 trials. The starting hand was also counterbalanced between participants. If participants did not respond within $800 \mathrm{~ms}$ on Go trials, the Go stimulus was replaced with the words "TOO SLOW" for $500 \mathrm{~ms}$, and then a black screen until the next trial began.

2.2.3. Restrained-Stop condition. In the Restrained-Stop task, white capital letters $\mathrm{F}$ and $\mathrm{P}$ were displayed for $800 \mathrm{~ms}$ in the centre of a black screen with an SOA of $1500 \mathrm{~ms}$ mean (range 1200-1800 ms). Participants were instructed to respond to one letter with the left index finger on the left button ('A' on a standard QWERTY keyboard), and to the other letter with the right index finger on the right button ('L'), and to stop their response if the letter turned red. The assignment of letters to the left and right hand was counterbalanced between participants, and stop-signals were presented on $25 \%$ of trials. Participants first completed a practice block with 10 stimuli to practice responding to the Go letters, followed by a second practice block where the stop signal was introduced. The second practice block had 32 trials, with 8 stop-signals occurring, one for each letter and each delay from $50 \mathrm{~ms}, 150 \mathrm{~ms}, 250 \mathrm{~ms}$ and $350 \mathrm{~ms}$ after the onset of the Go stimulus. Participants then completed a further four experimental blocks of 64 trials each. In the experimental blocks, the first stop-signal in each block was delivered at $250 \mathrm{~ms}$ post-Go stimulus, and the delay for the next stop-signal was adjusted based on the inhibition accuracy. If the participant successfully inhibited their response, the next stopsignal was delivered $50 \mathrm{~ms}$ later; if they were unsuccessful, the next stop-signal was delivered 50 ms earlier (i.e., the staircase method; Osman et al., 1986). If participants did not respond within $800 \mathrm{~ms}$ for no-stop-signal trials, the Go stimulus was replaced with the words "TOO SLOW" for $500 \mathrm{~ms}$, then a black screen until the next trial began. Similar restrained instructions were given as per Jones et al. (2011a,b), with participants instructed that successful inhibition of responses when the letters turn red was the most important task.

2.2.4. Combined condition. In the Combined task, white capital letters $\mathrm{F}$ and $\mathrm{P}$ were displayed for $800 \mathrm{~ms}$ in the centre of a black screen superimposed on images of water, with an SOA of 1500 ms mean (range 1200-1800 ms). Participants were instructed to respond to one letter with the left index finger, and to the other letter with the right index finger (with keys as above), and to stop their response if the background image changed to beer. The same four images of beer and water were used as in the Beer-NoGo condition and by Houben et al. (2011, 2012) and Bowley et al. (2013). All other task details, including the setting of stop-signal delay, were the same as for the Restrained-Stop task, with the exception that participants were instructed that fast responses and accurate inhibition were equally important, in line with the instructions that are typically given with the Stop-Signal task.

2.2.5. Brief alcohol intervention condition. An Australian BAI was completed online, consisting of demographic questions, questions from the Alcohol Use Disorders Identification Test (AUDIT; Saunders et al., 1993; see below for details), alcohol consump- tion patterns in the previous four weeks, the impact of other students' drinking, attitudes to alcohol labelling, and current smoking behaviours. At the completion of the BAI, they were provided with feedback and interpretation of their AUDIT score, peak blood alcohol content, money spent on alcohol per year, their drinking in relation to others of the same age and gender, suggestions on ways to reduce their drinking, and other information on support services available to them.

\subsection{Measures}

2.3.1. Questionnaires. Participants completed a short demographic questionnaire, the AUDIT (Saunders et al., 1993), the Barratt Impulsiveness Scale (BIS-11; Patton et al., 1995), and a Timeline Followback (TLFB) questionnaire (Sobell and Sobell, 1992).

The demographic questionnaire consisted of questions about age, sex, handedness, confirmation of eligibility criteria, and a question concerning thoughts about reducing drinking "Have you been concerned about your own drinking levels and considered cutting down?" with possible answers: No (66\% of participants); Yes, but not in the last year (9\%); and Yes, during the last year (25\%). These options were evenly distributed between conditions $\left(\chi^{2}=4.133\right.$, $\mathrm{df}=8, \mathrm{p}=0.845)$. Recoded as a Yes (34\%) or No (66\%) answer, intention to reduce drinking was included as a factor in preliminary analyses but was not found to significantly alter the results. This question will therefore not be discussed further.

The AUDIT consists of 10 multiple choice questions and assesses the domains of alcohol consumption, drinking behavior, and alcohol-related problems. Total scores range from 0 to 40; a total score of 8 or more indicates hazardous and harmful drinking, while a score of 20 or more indicates probable dependence (Babor et al., 2001).

The BIS- 11 consists of 30 questions indexing attentional impulsiveness, motor impulsiveness and non-planning impulsiveness. Total scores can range between 30 and 120, with an average for undergraduate students of 63.8, and for substance abusers 69.3 (Patton et al., 1995).

For the TLFB (Sobell and Sobell, 1992), participants were asked to record the number of standard drinks of beer, table wine, spirits, and fortified wine they consumed on each of the last several days. Participants completed this questionnaire twice; at the first session, they were asked to complete the record regarding the week before entering the lab, while at the second session the reference period was the week between experimental sessions. Participants were asked to reference an Australian standard drinks guide throughout completion.

\subsubsection{Taste test. As a measure of immediate alcohol consumption,} participants completed a bogus taste test of beer and soft drink. Participants were presented with $330 \mathrm{~mL}$ of orange-flavoured soft drink and $330 \mathrm{~mL}$ of non-alcoholic beer ( $0.3 \%$ alcohol by volume) in clear unlabeled plastic cups, and asked to rate their current level of thirst on a 7-point Likert scale ranging from $1=$ Not at all thirsty to $7=$ Thirsty, and then "consume as much or as little of each drink" as they wished in order to rate the beer and soft drink on four 7-point scales (Pleasant-Unpleasant, Flat-Gassy, Bitter-Sweet, and Tasteless-Strong-tasting). They were informed that they could rate them in any order, and that they had five minutes to make their ratings, but could stop earlier if they wished. The experimenter then closed the door to the laboratory and started the timer. After the experimental session was complete and the participant had left the laboratory, the experimenter noted the time taken on the taste test, and measured the amount of beer and soft drink consumed. Participants were not informed that the beer was non-alcoholic, nor that the amount consumed would be measured. Jones et al. (2016a) 
have recently published a review of the validity of the taste test for these purposes.

2.3.3. Awareness probe. At the end of the study, participants completed a written open-ended questionnaire which probed for awareness of the study's aims, and whether an expectation of driving reduced alcohol consumption on the taste test. Only 5.3\% reported that they limited beer consumption on the taste test due to an expectation of driving later that day and wanting to avoid exceeding the legal blood alcohol concentration limit; these were evenly distributed between the conditions $\left(\chi^{2}=2.479, \mathrm{df}=4\right.$, $\mathrm{p}=0.648$ ), and exclusion of these participants did not alter taste test results. No participants identified the correct and specific purpose of the Control, Simple-Stop or Restrained-Stop tasks; but 5 participants in the BAI condition and 1 in the Beer-NoGo condition correctly identified the purpose of these tasks. However, exclusion of the participant in the Beer-NoGo condition did not alter results; comparisons between those who did and did not identify the purpose of the BAI also were not significantly different. Therefore, the results from the awareness probe will not be discussed further.

2.3.4. Implicit association task and flanker task. Participants also completed implicit association tasks (IAT; Greenwald et al., 1998) and flanker tasks (Eriksen and Eriksen, 1974) to assess changes in attitudes to beer and in inhibitory processing as possible mechanisms for our interventions. However, these measures showed only effects of time and no effects or interactions involving condition, and to save space, we present them as Supplementary material only.

\subsection{Procedure}

On arrival at the first session (60 min), the experimental procedure was briefly explained (in line with the cover story that the study investigated the relationship between attitudes and preferences for beer, and reaction time on computer tasks) before participants gave consent to participate. Participants next completed, in order, a brief demographics questionnaire, the AUDIT, the $\mathrm{BIS}$, and a questionnaire on alcohol consumption over the past week using the TLFB as described above. Next participants completed, in order, the IAT and flanker tasks, then their assigned intervention condition task or the online BAI. Following this, participants again completed, in order, the IAT, the flanker task, and the taste test.

One week later at the second session (30 min), participants completed, in order, the TLFB for alcohol consumption in the week between sessions, the IAT, the flanker task, and the awareness probe questionnaire. Next, participants were fully debriefed on the real aims of the study and the purpose of each measure, before a second consent to participate was sought. No participant refused consent after debriefing. Lastly, participants received their \$25 reimbursement or course points along with gender-specific information on their alcohol consumption relative to Australian health guidelines and relative to others aged 18 years and over, and information about how to reduce alcohol consumption.

\subsection{Data analysis}

The total AUDIT and BIS scores were calculated according to standard scoring procedures for those questionnaires (Patton et al., 1995; Saunders et al., 1993).

TLFB data was subject to several calculations, to capture subtle changes in risky drinking behaviours; for example, two participants may each consume a total of 14 standard drinks per week, but the riskiness of drinking is greater if those 14 drinks are consumd in a single episode, compared to an average of 2 drinks per day. Therefore, in addition to the primary measure of total number of standard drinks consumed per week, we calculated a number of secondary measures: number of standard drinks of beer consumed per week (in case the beer pictures presented in the Beer-NoGo and Combined tasks reduced beer consumption more than other drinks); number of days on which some alcohol was consumed per week (drinking days/week); number of days on which more than four standard drinks were consumed (binge episodes/week), number of standard drinks consumed on the heaviest drinking day (maximum drinks/day); and average number of standard drinks consumed on days when drinking occurred (average drinks/drinking day). Australian alcohol consumption guidelines (National Health and Medical Research Council, 2009) advise against consuming more than four standard drinks on one occasion, and are identical for males and females; therefore we set the same binge definitions for males and females. These measures were calculated for both sessions, and due to non-normal distributions, log scores were calculated for all measures (base 10, with 1 added to all scores to avoid taking the log of zero).

Taste test consumption was analysed both as the total amount of beer consumed, as well as the amount of beer consumed as a percentage of total liquid consumed.

For completeness, we also calculated the error rates and reaction time (RT) for Go and NoGo trials for the Control and Beer-NoGo conditions, and Go RT and stop-signal reaction time (SSRT; Logan et al., 1984) for the Restrained-Stop and Combined conditions.

\subsection{Statistical analysis}

Demographic data on categorical scales were subjected to a chisquare test. Demographic information on continuous scales, and scores on the AUDIT and BIS were subjected to separate one-way ANOVAs with Condition as a between subjects factor. If effects involving Condition were significant, we examined contrasts on the Condition factor comparing the Control condition with each other condition separately using Dunnett's test.

Weekly alcohol consumption measures (total drinks, beer drinks, drinking days/week, binge episodes/week, maximum drinks/day and average drinks/drinking day) were subjected to a mixed design ANOVA (with within subjects factor Time: baseline or follow-up, and contrasts on the Condition factor as above).

Taste test consumption variables (absolute amounts of beer and soft drink consumed, and beer consumed as a percentage of total fluid consumption) were subjected to planned analyses of covariance with time of testing as a covariate (it was expected from the outset that participants may drink less beer in the early hours of the day). Contrasts on the Condition factor were as above.

\section{Results}

Table 1 displays demographic information and behavioural task performance for each of the conditions. Participants assigned to each condition were well-matched on sex ratio $\left(\chi^{2}=1.188, \mathrm{df}=4\right.$, $\mathrm{p}=0.880$; sex was included as a factor in preliminary analyses but was not found to significantly alter the results), proportion of right-handed participants $\left(\chi^{2}=7.972, \mathrm{df}=4, \mathrm{p}=0.093\right)$, time of testing $(F(4,109)=0.474, p=0.755)$, age $(F(4,109)=0.202$, $\mathrm{p}=0.937)$, BIS-11 score $(\mathrm{F}(4,109)=0.617, \mathrm{p}=0.652)$ and AUDIT score $(F(4,109)=1.165, p=0.330)$. Participants in the four computerised task conditions performed their tasks as expected, with few omission errors for the Control and Beer-NoGo tasks, greater commission errors in the Beer-NoGo task, and SSRT in the normal healthy adult range (200-250 ms; Schmajuk et al., 2006) for the Restrained-Stop and Combined tasks. 
Table 1

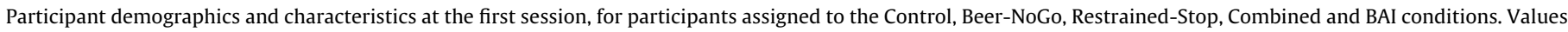
are mean (SE).

\begin{tabular}{|c|c|c|c|c|c|}
\hline & Control $(\mathrm{n}=22)$ & Beer-NoGo $(n=24)$ & Restrained-Stop $(\mathrm{n}=22)$ & Combined $(\mathrm{n}=22)$ & $\mathrm{BAI}(\mathrm{n}=24)$ \\
\hline Sex ratio (F:M) & 9:13 & $9: 15$ & $6: 16$ & $9: 13$ & $9: 15$ \\
\hline Right-handedness (\%) & 77.3 & 91.7 & 100.0 & 95.5 & 91.7 \\
\hline \multirow{2}{*}{ Age (years) } & 22.2 & 21.6 & 21.7 & 21.4 & 21.6 \\
\hline & $(0.8)$ & $(0.6)$ & $(0.7)$ & $(0.6)$ & $(0.6)$ \\
\hline \multirow[t]{2}{*}{ Time of testing ( $24 \mathrm{~h})$} & 13.4 & 13.9 & 13.7 & 13.0 & 13.6 \\
\hline & $(0.5)$ & $(0.5)$ & $(0.6)$ & $(0.5)$ & $(0.4)$ \\
\hline \multirow[t]{2}{*}{ BIS- 11 total } & 60.2 & 63.2 & 64.1 & 63.4 & 61.5 \\
\hline & $(2.0)$ & $(1.9)$ & $(2.1)$ & $(2.0)$ & $(2.1)$ \\
\hline \multirow{2}{*}{ AUDIT } & 11.4 & 12.0 & 12.0 & 9.6 & 11.4 \\
\hline & $(0.9)$ & $(1.0)$ & $(1.0)$ & $(0.7)$ & $(0.9)$ \\
\hline \multirow[t]{2}{*}{ Commission errors (\%) } & 0.6 & 9.0 & & & \\
\hline & $(0.2)$ & $(1.5)$ & & & \\
\hline \multirow[t]{2}{*}{ Omission errors (\%) } & 0.9 & 0.8 & & & \\
\hline & $(0.4)$ & $(0.4)$ & & & \\
\hline \multirow[t]{2}{*}{ Go RT (ms) } & 425.3 & 379.6 & 524.2 & 574.6 & \\
\hline & (10.3) & $(7.6)$ & (15.2) & (11.7) & \\
\hline \multirow[t]{2}{*}{ SSRT (ms) } & & & 204.7 & 256.6 & \\
\hline & & & (13.0) & (13.0) & \\
\hline
\end{tabular}

\subsection{Weekly alcohol consumption}

Fig. 1 shows the weekly alcohol consumption measures for participants in each condition, and for exploratory purposes, we also include in Table 2 the repeated measures effect size $\left(d_{R M}\right)$, defined as the difference between baseline and follow-up scores divided by the standard deviation of baseline scores, calculated separately for each condition; the effectiveness of one intervention condition relative to another is calculated by simple subtraction, denoted $\mathrm{d}_{\mathrm{IGPP}}$ (effect size for independent groups, pre-test post-test design; Becker, 1988; Morris and DeShon, 2002). Interpretation of these effect sizes follows Cohen's (1992) convention: $0.2=$ small, $0.5=$ medium, $0.8=$ large.

For total drinks per week, there was a significant reduction in consumption from baseline to follow-up $(F(1,109)=8.062$, $p=0.005)$, but there was no main effect of condition $(\mathrm{F}(4,109)=1.010, \mathrm{p}=0.405)$ or interaction of time and condition $(\mathrm{F}(4,109)=0.213, \mathrm{p}=0.931)$.

Beer standard drinks per week showed a significant reduction over time $(F(1,109)=10.963, p=0.001)$. There was no significant main effect of condition $(F(4,109)=1.232, p=0.302)$, although a significant condition $\mathrm{x}$ time interaction was observed $(F(4,109)=3.296, p=0.014)$. Specifically, participants in the BAI condition reduced their beer consumption more than Controls, although the effect only approached significance $(\mathrm{p}=0.064)$.

Drinking days per week showed no significant effect of time overall $(F(1,109)=2.396, p=0.125)$, and no significant main effect of condition $(F(4,109)=1.677, p=0.161)$. However, the condition $x$ time interaction was significant $(F(4,109)=2.553, \mathrm{p}=0.043)$. Specifically, participants in the BAI condition reduced their number of drinking days significantly more than Controls $(p=0.024)$.

Binge episodes per week showed a significant reduction between sessions $(F(1,109)=7.722, p=0.006)$, but there was no significant main effect of condition $(F(4,109)=0.190, p=0.943)$ or interaction $(\mathrm{F}(4,109)=0.552, \mathrm{p}=0.698)$.

The maximum number of standard drinks consumed on one day decreased significantly between sessions $(F(1,109)=7.938$, $\mathrm{p}=0.006$ ), but there was no significant main effect of condition $(\mathrm{F}(4,109)=0.809, \mathrm{p}=0.522)$ and no interaction $(\mathrm{F}(4,109)=0.056$, $\mathrm{p}=0.994)$.

Lastly, the average number of drinks consumed on a drinking day decreased between sessions $(F(1,108)=4.739, p=0.032)$, but there was no significant main effect of condition $(F(4,109)=1.018$, $\mathrm{p}=0.402)$ and no interaction $(\mathrm{F}(4,109)=0.852, \mathrm{p}=0.495)$.

\subsection{Taste test consumption}

Table 3 shows assorted taste test consumption measures by condition. There were no significant differences between conditions in the rating of thirst prior to the taste test $(F(4,109)=0.748$, $\mathrm{p}=0.562$ ), or in the time taken to finish the taste test $(3.3 \mathrm{~min}$; $\mathrm{F}(4,109)=0.105, \mathrm{p}=0.981)$. There were also no differences in the ratings of pleasantness of beer $(F(4,109)=1.581, p=0.185)$ or soft $\operatorname{drink}(\mathrm{F}(4,109)=1.166, \mathrm{p}=0.330)$.

For the absolute amount of beer consumed, time of testing was a significant covariate $(F(1,108)=4.658, p=0.033)$, such that participants consumed less beer earlier in the day. However, there were no differences between conditions in beer consumed $(F(4,108)=1.341$, $\mathrm{p}=0.259$ ).

For the absolute amount of soft drink consumed, time of testing was again a significant covariate $(F(1,108)=9.056, p=0.003)$, with less consumption earlier in the day. However, there were no differences between conditions in adjusted means $(F(4,108)=0.425$, $\mathrm{p}=0.790$ ).

For the relative amount of beer consumed (beer as a proportion of total fluid consumed), time of testing was not a significant covariate $(F(1,108)=1.113, p=0.297)$, and thus was removed from the model. A significant difference was observed between conditions $(\mathrm{F}(4,109)=2.930, \mathrm{p}=0.024)$; specifically, participants in the Combined condition consumed a significantly greater proportion of beer compared to Controls $(\mathrm{p}=0.049)$.

\section{Discussion}

We aimed to test whether training on an established or new version of inhibitory task produced significant reductions in alcohol consumption, after accounting for the effect of simple assessment of drinking. Our primary outcome measures were alcohol consumption in the week after compared to the week before the inhibitory training/intervention, and in an immediate postintervention bogus taste test.

The sample recruited was appropriate for the research question. The drinking habits of young adult university students are similar to the general population of the same age (Reavley et al., 2011), where risky drinking is prevalent (Australian Institute of Health and Welfare, 2014), and the AUDIT scores indicate that we obtained a sample of hazardous and harmful drinkers who are likely to benefit from reductions in alcohol consumption. Participants in the five conditions were also similar demographically, and had impulsivity (BIS-11) scores that were in the normal range for university 
Table 2

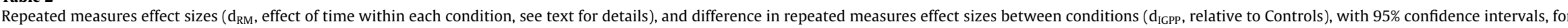
measures of weekly alcohol consumption. Confidence intervals that do not include zero are indicated with bold text.

\begin{tabular}{|c|c|c|c|c|c|c|c|}
\hline & & Control & Beer-NoGo & Restrained-Stop & Combined & BAI & All participants \\
\hline \multirow[t]{4}{*}{ Total standard drinks/week } & $d_{R M}$ & 0.336 & 0.301 & 0.212 & 0.291 & 0.472 & 0.324 \\
\hline & $(95 \% \mathrm{CI})$ & $(-0.052,0.723)$ & $(-0.112,0.714)$ & $(-0.138,0.562)$ & $(-0.051,0.632)$ & $(-0.107,1.052)$ & $(0.130,0.519)$ \\
\hline & $\mathrm{d}_{\mathrm{IGPP}}$ & & -0.035 & -0.123 & -0.045 & 0.137 & \\
\hline & $(95 \% \mathrm{CI})$ & & $(-0.601,0.531)$ & $(-0.645,0.399)$ & $(-0.561,0.471)$ & $(-0.560,0.834)$ & \\
\hline \multirow[t]{4}{*}{ Beer standard drinks/week } & $\mathrm{d}_{\mathrm{RM}}$ & 0.232 & 0.414 & 0.068 & -0.031 & 1.095 & 0.336 \\
\hline & $(95 \% \mathrm{CI})$ & $(-0.030,0.493)$ & $(0.075,0.753)$ & $(-0.305,0.442)$ & $(-0.376,0.314)$ & $(0.517,1.672)$ & $(0.162,0.509)$ \\
\hline & $\mathrm{d}_{\mathrm{IGPP}}$ & & 0.183 & -0.163 & -0.263 & 0.863 & \\
\hline & $(95 \% \mathrm{CI})$ & & $(-0.245,0.610)$ & $(-0.619,0.293)$ & $(-0.696,0.170)$ & $(0.230,1.496)$ & \\
\hline \multirow[t]{4}{*}{ Drinking days/week } & $\mathrm{d}_{\mathrm{RM}}$ & -0.173 & 0.246 & 0.187 & -0.115 & 0.571 & 0.143 \\
\hline & $(95 \% \mathrm{CI})$ & $(-0.423,0.077)$ & $(-0.117,0.610)$ & $(-0.156,0.530)$ & $(-0.418,0.189)$ & $(0.088,1.053)$ & $(-0.020,0.306)$ \\
\hline & $\mathrm{d}_{\mathrm{IGPP}}$ & & 0.420 & 0.360 & 0.058 & 0.744 & \\
\hline & $(95 \% \mathrm{CI})$ & & $(-0.022,0.861)$ & $(-0.064,0.785)$ & $(-0.335,0.452)$ & $(0.201,1.288)$ & \\
\hline \multirow[t]{4}{*}{ Binge episodes/week } & $d_{R M}$ & 0.048 & 0.340 & 0.443 & 0.272 & 0.199 & 0.268 \\
\hline & $(95 \% \mathrm{CI})$ & $(-0.308,0.404)$ & $(-0.069,0.748)$ & $(0.053,0.833)$ & $(-0.095,0.638)$ & $(-0.237,0.635)$ & $(0.091,0.445)$ \\
\hline & $\mathrm{d}_{\mathrm{IGPP}}$ & & 0.292 & 0.395 & 0.224 & 0.151 & \\
\hline & $(95 \% \mathrm{CI})$ & & $(-0.250,0.834)$ & $(-0.133,0.924)$ & $(-0.287,0.735)$ & $(-0.412,0.715)$ & \\
\hline \multirow[t]{4}{*}{ Maximum standard drinks/day } & $\mathrm{d}_{\mathrm{RM}}$ & 0.414 & 0.367 & 0.212 & 0.455 & 0.292 & 0.341 \\
\hline & $(95 \% \mathrm{CI})$ & $(-0.119,0.948)$ & $(-0.044,0.777)$ & $(-0.206,0.631)$ & $(0.012,0.898)$ & $(-0.273,0.858)$ & $(0.129,0.553)$ \\
\hline & $\mathrm{d}_{\mathrm{IGPP}}$ & & -0.047 & -0.202 & 0.041 & -0.122 & \\
\hline & $(95 \% \mathrm{CI})$ & & $(-0.720,0.626)$ & $(-0.880,0.476)$ & $(-0.653,0.734)$ & $(-0.899,0.656)$ & \\
\hline \multirow[t]{4}{*}{ Average drinks/drinking day ${ }^{a}$} & $\mathrm{~d}_{\mathrm{RM}}$ & 0.459 & 0.138 & 0.100 & 0.460 & -0.036 & 0.209 \\
\hline & $(95 \% \mathrm{CI})$ & $(-0.042,0.960)$ & $(-0.224,0.501)$ & $(-0.267,0.467)$ & $(0.048,0.871)$ & $(-0.484,0.412)$ & $(0.025,0.392)$ \\
\hline & $\mathrm{d}_{\mathrm{IGPP}}$ & & -0.321 & -0.359 & 0.001 & -0.495 & \\
\hline & $(95 \% \mathrm{CI})$ & & $(-0.939,0.298)$ & $(-0.980,0.262)$ & $(-0.648,0.649)$ & $(-1.167,0.177)$ & \\
\hline
\end{tabular}

$\mathrm{d}_{\mathrm{RM}}$ : Positive effect size represents a reduction in drinking from baseline to follow-up. $\mathrm{d}_{\mathrm{IGPP}}$ : Positive effect size represents a greater reduction in the test condition relative to Controls.

${ }^{a} \mathrm{~N}=23$ for the BAI condition, and $\mathrm{N}=113$ for All Participants, since one participant had zero drinking days in the week after the intervention. 

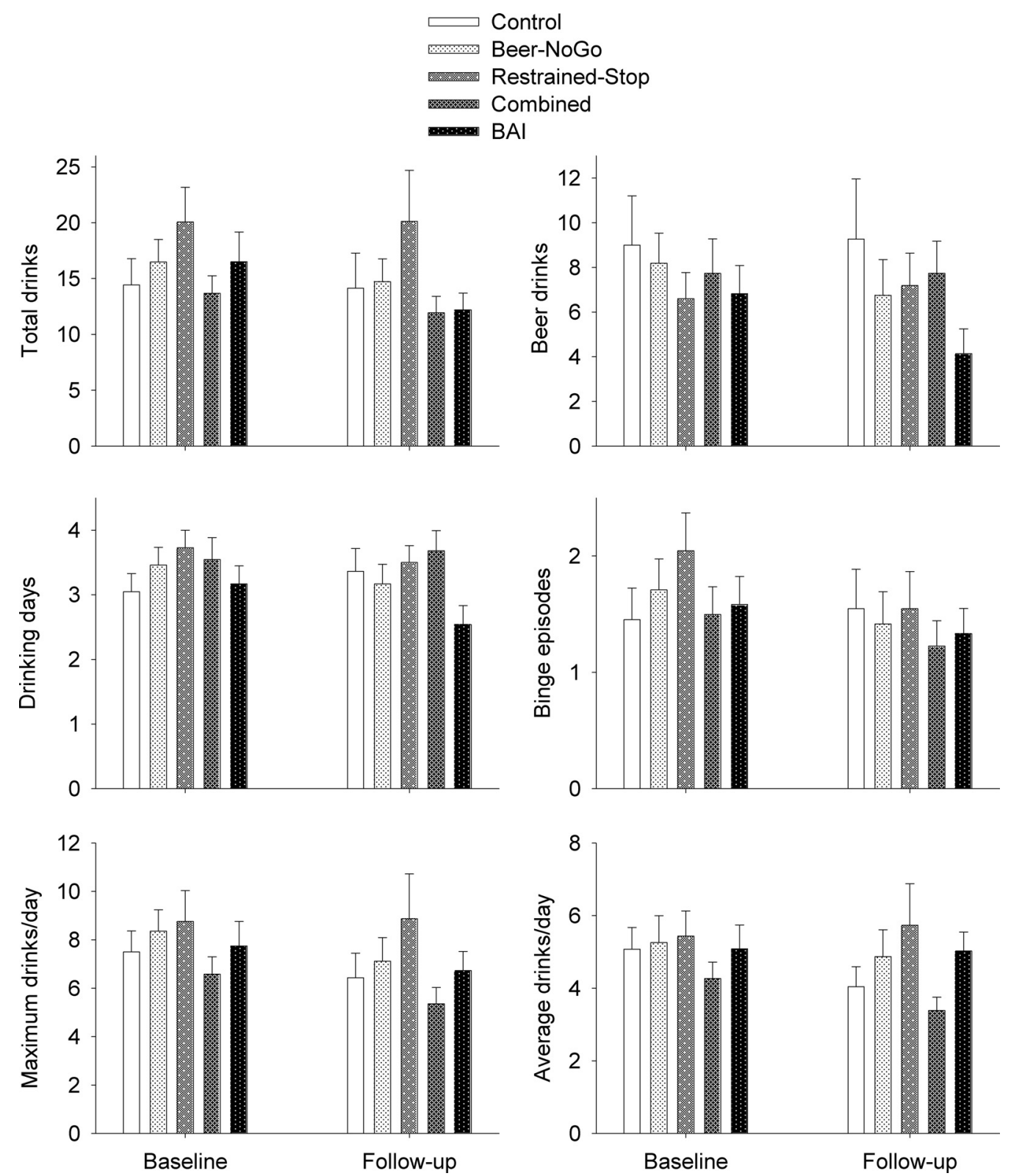

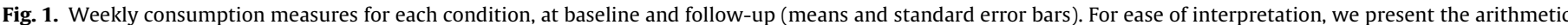

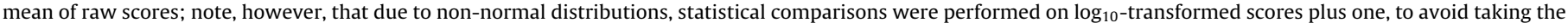
$\log$ of zero.

Table 3

Taste test consumption measures for each group. Values are mean (SE).

\begin{tabular}{|c|c|c|c|c|c|}
\hline & Control & Beer-NoGo & Restrained-Stop & Combined & BAI \\
\hline \multirow{2}{*}{ Thirst $^{\mathrm{a}}$} & 5.1 & 4.6 & 5.1 & 5.0 & 4.8 \\
\hline & $(0.3)$ & $(0.2)$ & $(0.3)$ & $(0.2)$ & $(0.2)$ \\
\hline \multirow[t]{2}{*}{ Time taken (mins) } & 3.3 & 3.2 & 3.3 & 3.4 & 3.3 \\
\hline & $(0.3)$ & $(0.2)$ & $(0.3)$ & $(0.3)$ & $(0.3)$ \\
\hline \multirow[t]{2}{*}{ Beer pleasantness } & 3.4 & 2.9 & 3.0 & 2.5 & 3.4 \\
\hline & $(0.4)$ & $(0.3)$ & $(0.3)$ & $(0.2)$ & $(0.3)$ \\
\hline \multirow{2}{*}{ Soft drink pleasantness } & 3.2 & 3.3 & 2.7 & 3.0 & 2.5 \\
\hline & $(0.4)$ & $(0.3)$ & $(0.3)$ & $(0.3)$ & $(0.3)$ \\
\hline \multirow[t]{2}{*}{ Beer consumed $(\mathrm{mL})^{\mathrm{b}}$} & 128.0 & 148.6 & 133.5 & 194.0 & 165.6 \\
\hline & $(23.1)$ & $(22.1)$ & $(23.1)$ & $(23.2)$ & $(22.1)$ \\
\hline \multirow{2}{*}{ Soft drink consumed $(\mathrm{mL})^{\mathrm{b}}$} & 131.2 & 102.9 & 132.7 & 108.0 & 121.2 \\
\hline & $(20.9)$ & $(20.1)$ & $(20.9)$ & $(21.0)$ & $(20.0)$ \\
\hline \multirow[t]{2}{*}{ Beer consumed (\% total fluid) } & 51.1 & 59.4 & 47.4 & 66.0 & 55.8 \\
\hline & $(4.5)$ & $(4.5)$ & $(4.2)$ & (3.9) & (3.7) \\
\hline
\end{tabular}

a $n=21$ for Control condition. Thirst ratings: $1=$ not at all thirsty, $7=$ thirsty. Pleasantness ratings: $1=$ pleasant, $7=$ unpleasant.

b Means are presented after adjusting for the covariate, time of testing. 
undergraduates (Patton et al., 1995) and also not different between conditions.

For weekly consumption, a significant reduction over time (baseline vs. follow-up) was observed across participants for our primary measure of total drinks per week, as well as several secondary measures of consumption. Examination of Table 2 (last column) indicates that the within-subjects effect sizes across all participants are in the range $0.21-0.34$; that is, participation in this study was associated with a small-medium reduction in various measures of weekly consumption, regardless of the condition/task to which the participant was randomly assigned. This is in line with many other studies reporting an effect of assessment on alcohol consumption (Bartsch et al., 2016; Gallen, 1974; Kypri et al., 2007; McCambridge and Day, 2008; McCambridge and Kypri, 2011; see Clifford and Maisto, 2000, for a review). As McCambridge and Day (2008) point out, it is not clear whether reductions in consumption are due to the assessment questions inciting reflections about drinking behaviours, and therefore change in those behaviours, or whether it is due to the knowledge that consumption was being monitored generally (a Hawthorne effect; Mayo, 1933; Roethlisberger and Dickson, 1939). Although participants were told that the study investigated the relationship between reaction time and attitudes and preferences for beer, they were informed at the beginning of the first session, prior to entry to the study, that there would be questions about alcohol consumption, and at the end of the first session that the second session would involve some of the same questions from the first session. It is also unclear how much of the self-reported reduction in alcohol consumption represents a real reduction and how much a bias towards giving socially desirable responses (i.e., lower consumption). It is also possible that the reduction in drinking is not due specifically to questionnaire assessment of alcohol consumption, but rather to other experimental factors which were common across conditions. For example, during debriefing, several participants (incorrectly) identified the IAT as an experimental variable to manipulate drinking, specifically pointing to the link between negative words and images of beer in that task (see Supplementary material). Although images of beer were in fact presented equally as often with positive and negative words, and the order of these pairings was counterbalanced between participants, we cannot rule out that the IAT may have had some unintended non-specific effect on alcohol consumption across the conditions. Similarly, participants completed the Eriksen flanker task twice in the first session, and practice on that task may have primed a more cautious response set for all participants, as per Jones et al. (2011a,b). From a public health perspective, the mechanism of this reduction in consumption is unimportant so long as it is reliable (McCambridge and Day, 2008), and suggests the utility of widespread application of these protocols via the internet or in primary care settings for lowering alcohol consumption at the population level (McCambridge and Day, 2008).

However, the original question remains whether inhibitory training can produce reliable reductions in drinking. In previous studies, the control conditions have linked alcohol with speeded response execution (Bowley et al., 2013; Houben et al., 2011, 2012), such that the Beer-Go condition has been associated with slightly increased weekly alcohol consumption (Houben et al., 2011, 2012), or have primed impulsive responding via instructions that speed is more important than accuracy (Jones et al., 2011a,b), resulting in increased taste test consumption. Thus, the differences between conditions observed in previous studies may have been due to increases in the 'control' condition as much as decreases in the inhibitory training conditions. In the current study, our Control task neither required inhibition (responses required on only $25 \%$ of trials, and therefore the dominant response is to do nothing on NoGo trials), nor did it favour impulsive responding or link alcohol to response execution. Under these conditions, participants in the
Control condition showed small-medium reductions in consumption in line with the non-specific effect discussed above; however, neither the Beer-NoGo, nor Restrained-Stop, nor Combined task produced significantly greater reductions in consumption relative to the Control condition. Since we did not include a Beer-Go or Disinhibited-Stop condition, we cannot conclusively confirm that these conditions are associated with a significant increase in consumption relative to Controls. However, from the current results, it is possible that any apparent reductions associated with inhibitory tasks reported in previous research were either an artefact of a poorly chosen comparison condition which increased consumption, or due to an effect of research participation more than a true intervention effect. More work directly comparing, for example, Beer-Go, Beer-NoGo and Control conditions may help to confirm this speculation.

In contrast to the inhibitory tasks, the BAI condition was successful at reducing the number of standard drinks of beer per week, and the number of drinking days per week, relative to Controls. The BAI also had the largest effect size for total drinks per week, although not significantly greater than the Control condition. This is in line with numerous prior studies showing that the BAI is effective at reducing alcohol consumption among heavy drinkers (Bertholet et al., 2005; Kypri et al., 2009; Samson and Tanner-Smith, 2015).

For the taste test (Jones et al., 2016a), we expected to find reductions in consumption for all inhibitory tasks and the BAI relative to Controls, in line with previous research (Bowley et al., 2013; Houben et al., 2011; Jones et al., 2011a,b; Jones and Field, 2013). However, contrary to expectations, we observed no significant difference in consumption for participants in the Beer-NoGo, Restrained-Stop and BAI conditions, and an increase in consumption for the Combined condition, although it is possible that this was due to an increased liking for the beer, as pleasantness ratings were slightly greater in this group (see Table 3). Unlike the pre-post measures used for weekly consumption, it is difficult to estimate the size of the Hawthorne effect for taste test consumption in the Control condition; that is, participants knew they were participating in a research study, even if they were unaware of the measures or hypotheses under consideration, and it is impossible to estimate how much they would have consumed under different circumstances. However, the similar consumption in the Beer-NoGo and Restrained-Stop interventions mirrors the results for weekly consumption in suggesting that the effect of these interventions on reducing alcohol consumption is small.

Several limitations of the current research are apparent. Firstly, we matched the probability of inhibitory signals in the Beer-NoGo task to the probability (25\%) in the Restrained-Stop and Combined task; this was intended not only to ease comparisons between tasks but also would make inhibition more difficult than the equiprobable Beer-NoGo tasks previously used (Bowley et al., 2013; Houben et al., 2011, 2012). However, the consequence of halving the number of inhibition trials was that it also halved the opportunities for participants to learn the contingencies between alcohol images and inhibition. It is possible that this affected the results for the BeerNoGo condition, but of course does not explain the failure of the Restrained-Stop task to produce significant reductions in drinking, since the Restrained-Stop was a replication of previous research (Jones et al., 2011a,b).

Secondly, the training conditions differed on the use of alcohol images; the Beer-NoGo and Combined tasks presented beer images during training, while the Control and Restrained-Stop tasks did not. It is possible that exposure to alcohol images increases alcohol consumption (e.g., by increasing craving; Papachristou et al., 2012), thereby counteracting any reduction associated with pairing these images with inhibition. It may be necessary in future research to test the effect of simply presenting alcohol images with no associated task to determine the extent of subsequent alterations in 
alcohol consumption, and thereby assist with estimating the specific effect of pairing alcohol images with inhibition.

Thirdly, inhibitory training is one form of cognitive bias modification, widely believed to be effective in behaviour modification studies (e.g., Clarke et al., 2014; MacLeod and Clarke, 2015; but see Cristea et al., 2016; Field et al., 2016; Wiers, 2016 for debate). However, a recent meta-analysis (MacLeod and Grafton, 2016) has shown that a change in bias is critical for achieving a change in behaviour. In the current study, we did not specifically test associations between alcohol cues and stopping behaviour, such as slowing of Go RT on alcohol catch trials or decreases in commission error rate over the course of training (see Jones and Field, 2013). Because we did not test for a change in alcohol-stop associations, it is impossible to know whether the inhibitory training paradigms successfully altered the bias, and therefore whether a change in behaviour should be expected.

Fourthly, we have used the term 'inhibitory training' here for consistency with previous research; however, in the broader learning literature, 'training' typically involves multiple sessions with progressing task difficulty and feedback on performance (e.g., Green and Bavelier, 2008). In contrast, participants in the present study completed only a single brief session of ICT, which did not become progressively more difficult over time. The aim of this study was to improve our understanding of the mechanism of action of ICT on short-term drinking behaviour rather than demonstrate its efficacy as a treatment intervention for alcohol use disorders. Thus, experiments such as the present study should be seen as a form of 'experimental medicine' in contrast to randomised controlled trials (RCTs) that evaluate efficacy of interventions (see Sheeran et al., 2017). We await findings from ongoing RCTs of multiple sessions of ICT for the reduction of alcohol consumption in heavy drinkers who are motivated to cut down (e.g., Jones et al., 2014; van Deursen et al., 2013).

Lastly, it is possible that our lack of significant interactions between condition and time is due to a lack of statistical power (as a result of inadequate sample size) rather than a real null effect. It was difficult to predict a priori what would be the repeated measures effect size for the Control condition, since it is novel and had not been tested before. In this respect, the study could be considered a preliminary investigation, with an appropriately small pilot sample size. We included estimates of the effect sizes for all weekly consumption measures in Table 2; while most of the point estimates of effect size are only small- to medium-sized, and most of the $95 \%$ confidence intervals for $d_{R M}$ contain zero (indicating a non-significant effect), many of the confidence intervals also have quite large positive upper limits (meaning, for example, that the true $d_{R M}$ for weekly alcohol consumption could be as large as $0.5-0.7$ for the inhibitory and control tasks, a substantial and possibly clinically relevant reduction). The problem is further compounded when we consider the size of the confidence intervals surrounding $\mathrm{d}_{\mathrm{IGPP}}$, which, for total weekly consumption, range across conditions from medium negative effects to medium-large positive effects (Hoenig and Heisey, 2001). Clearly, replication with considerably larger sample sizes will be required to further explore and confirm or reject the null result reported here (Button et al., 2013); the effect sizes reported here will aid researchers to plan future studies of inhibitory training more generally. However, we consider it necessary to publish studies with null effects as well as those with significant effects, in order that the true utility of these tasks can be estimated.

Despite the discouraging lack of a large effect of inhibitory training, we do not consider that investigation of inhibitory training as a means to reduce alcohol consumption is a fruitless endeavor for future investigations. Rather, investigators will need to carefully consider the possible sources of any observed alterations in drinking behavior and ensure that the chosen inhibitory task design produces an effect beyond that of simple assessment, and indeed beyond the gold-standard BAI. In contrast to our hypotheses, the Combined task did not produce greater reductions than the established tasks. Contrary to our intentions that an alcohol image was the signal for inhibition, it is possible that participants instead initiated the stopping process when the image changed, and not to the image of alcohol in particular. A selective stop paradigm (e.g., De Jong et al., 1995) could be used to test this idea, such that a neutral background image (e.g., landscapes) might change to either an alcohol-related image (in which case the participants were required to withhold the button press) or a water-related image (in which case the participants ignore the signal and respond). We predict greater reductions would be observed in a group completing this form of training compared to a Control task, or to the Combined task as tested here. Similarly, our superposition of the Go/NoGo letter stimuli on water and beer images matches previous research using the Beer-NoGo task (Houben et al., 2011, 2012; Bowley et al., 2013), but we see no reason (apart from the desire to study catch trials as noted above) in future research not to discard the letter stimuli altogether and instruct participants to respond to water images and not respond to beer images, again with the goal of more directly linking alcohol with inhibition. With regard to the other tasks, in line with recent meta-analyses showing that Go/NoGo tasks produce stronger effects than Stop-Signal tasks (Allom et al., 2016; Jones et al., 2016b), our Beer-NoGo task did indeed produce slightly greater reductions in most measures of consumption than the Restrained-Stop condition (see Table 2). In light of this, we suggest that future research focuses on the Beer-NoGo task, and identifying not only the features of the task that produce large reductions in consumption, but also the mechanisms behind this reduction. We also point out that the Beer-NoGo task was the most effective of the inhibitory tasks at reducing the number of beer drinks per week; we speculate that this might be due to the specific pairing of beer with NoGo stimuli. We question whether the BeerNoGo task might be more effective with participants who preferred beer (rather than merely expressing a liking for beer, as here), or whether a more specific set of images of the participant's preferred drink (e.g., beer vs. red wine vs. white wine), or even preferred brand, might also be more effective at reducing consumption of specific drinks, than the less personally-relevant generic images of beer.

In summary, in the current study we examined whether established and new versions of inhibitory training produced substantial reductions in alcohol consumption, beyond the non-specific effect of simply taking part in alcohol research. However, neither the new version of inhibitory training, nor previously studied versions, produced significant reductions in drinking beyond that observed in the control condition. While our results do not necessarily suggest that inhibitory training should be abandoned as an intervention worthy of further research (and regardless, should be interpreted with caution due to concerns about low power to detect effects of training), they do highlight the need for consideration of the effects of simple assessment alone on alcohol consumption. Furthermore, the search continues for an inhibitory task variant which is more effective than the well-established Brief Alcohol Intervention.

\section{Conflict of interest}

No conflict declared.

\section{Contributors}

JS, SJ, KH and MF were responsible for the study concept and design. JS and ND completed data collection. JS drafted the manuscript. All authors provided critical revision of the manuscript 
for important intellectual content, and approved the final version for publication.

\section{Role of funding source}

This study was funded by an Australian Rotary Health Postdoctoral Research Fellowship to JS. The National Drug and Alcohol Research Centre at the University of NSW is supported by funding from the Australian Government under the Substance Misuse Prevention and Service Improvements Grants Fund. Funding sources had no role in the study design, collection, analysis or interpretation of data, in the decision to publish, or in the writing of the report.

\section{Acknowledgements}

Thanks are due to Mr Tony Kemp for writing the stimulus presentation programs, and Mr Andrew Jones for his comments on an earlier draft.

\section{Appendix A. Supplementary data}

Supplementary data associated with this article can be found, in the online version, at http://dx.doi.org/10.1016/j.drugalcdep.2016. 12.018 .

\section{References}

Allom, V., Mullan, B., Hagger, M., 2016. Does inhibitory control training improve health behaviour? A meta-analysis. Health Psychol. Rev. 10, 168-186.

American Psychiatric Association, 2013. DSM-5. Diagnostic and Statistical Manual of Mental Disorders, 5th edition. American Psychiatric Publishing, Arlington, VA.

Australian Institute of Health and Welfare, 2014. National Drug Strategy Household Survey Detailed Report 2013. Drug Statistics Series 28 Category Number PHE 183, Canberra.

Babor, T.F., Higgins-Biddle, J.C., Saunders, J.B., Monteiro, M.G., 2001. AUDIT: The Alcohol Use Disorders Identification Test: Guidelines For Use In Primary Health Care. World Health Organization, Geneva.

Barkley, R.A., 1997. Behavioural inhibition, sustained attention, and executive functions: constructing a unifying theory of ADHD. Psychol. Bull. 121, 65-94.

Bartsch, A.L., Kothe, E., Allom, V., Mullan, B., Houben, K., 2016. The effect of non-specific response inhibition training on alcohol consumption: an intervention. J. Addict. Res. Ther. 7, 260.

Becker, B.J., 1988. Synthesizing standardized mean-change measures. Br. J. Math Stat. Psychol. 41, 257-278.

Bertholet, N., Daeppen, J., Wietlisbach, V., Fleming, M., Burnand, B., 2005. Reduction of alcohol consumption by brief alcohol intervention in primary care: systematic review and meta-analysis. Arch. Intern. Med. 165, 986-995.

Best, M., Lawrence, N.S., Logan, G.D., McLaren, I.P.L., Verbruggen, F., 2016. Should I stop or should I go? The role of associations and expectancies. J. Exp. Psychol. Hum. Percept. Perf. 42, 115-137.

Bowditch, W.A., Verbruggen, F., McLaren, I.P.L., 2016. Associatively mediated stopping: training stimulus-specific inhibitory control. Learn. Behav. 44 162-174.

Bowley, C., Faricy, C., Hegarty, B.D., Johnstone, S.J., Smith, J.L., Kelly, P.J., Rushby, J.A., 2013. The effects of inhibitory control training on alcohol consumption, implicit alcohol-related cognitions and brain electrical activity. Int. J. Psychophysiol. 89, 342-348.

Button, K.S., Ioannidis, J.P.A., Mokrysz, C., Nosek, B.A., Flint, J., Robinson, E.S.J., Munafo, M.R., 2013. Power failure: why small sample size undermines the reliability of neuroscience. Nat. Rev. Neurosci. 14, 451-451.

Clarke, P.J.F., Notebaert, L., MacLeod, C., 2014. Absence of evidence or evidence of absence: reflecting on therapeutic implementations of attentional bias modification. BMC Psychiatry 14, 8-8.

Clifford, P.R., Maisto, S.A., 2000. Subject reactivity effects and alcohol treatment outcome research. J. Stud. Alcohol 61, 787-793.

Cohen, J., 1992. A power primer. Psychol. Bull. 112, 155-159.

Cristea, I.A., Kok, R.N., Cuijpers, P., 2016. The effectiveness of cognitive bias modification interventions for substance addictions: a meta-analysis. PLoS One 11, e0162226.

De Jong, R., Coles, M.G.H., Logan, G.D., 1995. Strategies and mechanisms in nonselective and selective inhibitory motor control. J. Exp. Psychol. Hum. Percept. Perf., 498-511.

Diamond, A., 2013. Executive functions. Annu. Rev. Psychol. 64, 135-168.

Eriksen, B.A., Eriksen, C.W., 1974. Effects of noise letters upon the identification of a target letter in a nonsearch task. Percept. Psychophys. 16, 143-149.

Field, M., Jones, A., Christiansen, P., 2016. Questionable classification of studies and choice of outcome measures. PLoS One 11, e0162226 (comment).
Gallen, M., 1974. Toward an understanding of follow-up research with alcoholics. Psychol. Rep. 34, 877-878.

Green, C.S., Bavelier, D., 2008. Exercising your brain: a review of human brain plasticity and training-induced learning. Psychol. Aging 23, 692-701.

Greenwald, A.G., McGhee, D.E., Jordan, L.K., 1998. Measuring individual differences in implicit cognition: the implicit association test. J. Pers. Soc. Psychol. 74, 1464-1480.

Hester, R., Lubman, D.I., Yücel, M., 2010. The role of executive control in human drug addiction. In: Self, D.W., Staley Gottschalk, J.K. (Eds.), Behavioral Neuroscience of Drug Addiction. Springer, Berlin, Heidelberg, pp. 301-318.

Hoenig, J.M., Heisey, D.M., 2001. The abuse of power: the pervasive fallacy of power calculations for data analysis. Am. Stat. 55, 1-6.

Houben, K., Nederkoorn, C., Wiers, R.W., Jansen, A., 2011. Resisting temptation: decreasing alcohol-related affect and drinking behavior by training response inhibition. Drug Alcohol Depend. 116, 132-136.

Houben, K., Havermans, R.C., Nederkoorn, C., Jansen, A., 2012. Beer à no-go: learning to stop responding to alcohol cues reduces alcohol intake via reduced affective associations rather than increased response inhibition. Addiction 107, 1280-1287.

Jentsch, J.D., Pennington, Z.T., 2014. Reward, interrupted: inhibitory control and its relevance to addictions. Neuropharmacology 76, 479-486.

Jones, A., Field, M., 2013. The effects of cue-specific inhibition training on alcohol consumption in heavy social drinkers. Exp. Clin. Psychopharmacol. 21, 8-16.

Jones, A., Cole, J., Goudie, A., Field, M., 2011a. Priming a restrained mental set reduces alcohol-seeking independently of mood. Psychopharmacology 218 557-565.

Jones, A., Guerrieri, R., Fernie, G., Cole, J., Goudie, A., Field, M., 2011b. The effects of priming restrained versus disinhibited behaviour on alcohol-seeking in social drinkers. Drug Alcohol Depend. 113, 55-61.

Jones, A., McGrath, E., Houben, K., Nederkoorn, C., Robinson, E., Field, M., 2014. A comparison of three types of web-based inhibition training for the reduction of alcohol consumption in problem drinkers: study protocol. BMC Public Health 14,796

Jones, A., Button, E., Rose, A.K., Robinson, E., Christiansen, P., Di Lemma, L., Field, M. 2016a. The ad-libitum alcohol 'taste test': secondary analyses of potential confounds and construct validity. Psychopharmacology 233, 917-924.

Jones, A., Di Lemma, L.C.G., Robinson, E., Christiansen, P., Nolan, S., Tudur-Smith, C., Field, M., 2016b. Inhibitory control training for appetitive behaviour change: a meta-analytic investigation of mechanisms of action and moderators of effectiveness. Appetite 97, 16-28.

Kypri, K., Langley, J.D., Saunders, J.B., Cashell-Smith, M.L., 2007. Assessment may conceal therapeutic benefit: findings from a randomized controlled trial for hazardous drinking. Addiction 102, 62-70.

Kypri, K., Hallett, J., Howat, P., McManus, A., Maycock, B., Bowe, S., Horton, N., 2009. Randomized controlled trial of proactive web-based alcohol screening and brief alcohol intervention for university students. Arch. Intern. Med. 169, $1508-1514$

Logan, G.D., Cowan, W.B., 1984. On the ability to inhibit thought and action: a theory of an act of control. Psychol. Rev. 91, 295-327.

Logan, G.D., Cowan, W.B., David, K.A., 1984. On the ability to inhibit simple and choice reaction time responses: a model and a method. J. Exp. Psychol. 10, $276-291$.

MacLeod, C., Clarke, P.J.F., 2015. The attentional bias modification approach to anxiety intervention. Clin. Psychol. Sci. 3, 58-78.

MacLeod, C., Grafton, B., 2016. Anxiety-linked attentional bias and its modification: illustrating the importance of distinguishing processes and procedures in experimental psychopathology research. Behav. Res. Ther. 86, 68-86.

Mayo, E., 1933. The Human Problems of An Industrial Civilization. MacMillan, New York.

McCambridge, J., Day, M., 2008. Randomized controlled trial of the effects of completing the alcohol use disorders identification test questionnaire on self-reported hazardous drinking. Addiction 103, 241-248.

McCambridge, J., Kypri, K., 2011. Can simply answering research questions change behaviour? Systematic review and meta analyses of brief alcohol intervention trials. PLoS One 6, e23748

Morris, S.B., DeShon, R.P., 2002. Combining effect size estimates in meta-analysis with repeated measures and independent-groups designs. Psychol. Method. 7, $105-125$.

National Health and Medical Research Council, 2009. Australian Guidelines To Reduce Health Risks From Drinking Alcohol, Canberra.

Nieuwenhuis, S., Yeung, N., van den Wildenberg, W., Ridderinkhof, K.R., 2003. Electrophysiological correlates of anterior cingulate function in a go/no-go task: effects of response conflict and trial type frequency. Cogn. Affect. Behav. Neurosci. 3, 17-26.

Noël, X., Van der Linden, M., d'Acremont, M., Bechara, A., Dan, B., Hanak, C., Verbanck, P., 2007. Alcohol cues increase cognitive impulsivity in individuals with alcoholism. Psychopharmacology 192, 291-298.

Osman, A., Kornblum, S., Meyer, D.E., 1986. The point of no return in choice reaction time: controlled and ballistic stages of response preparation. J. Exp. Psychol. Hum. Percept. Perf. 12, 243-258.

Papachristou, H., Nederkoorn, C., Havermans, R., van der Horst, M., Jansen, A., 2012. Can't stop the craving: the effect of impulsivity on cue-elicited craving for alcohol in heavy and light social drinkers. Psychopharmacology 219, 511-518.

Patton, J.H., Stanford, M.S., Barratt, E.S., 1995. Factor structure of the barrat impulsiveness scale. J. Clin. Psychol. 51, 768-774. 
Randall, W.M., Smith, J.L., 2011. Conflict and inhibition in the cued-Go/NoGo task Clin. Neurophysiol. 122, 2400-2407.

Reavley, N., Jorm, A., McCann, T., Lubman, D., 2011. Alcohol consumption in tertiary education students. BMC Public Health 11, 545

Roethlisberger, F.J., Dickson, W.J., 1939. Management and the Worker. Cambridge University Press, Cambridge, Massachusetts.

Samson, J.E., Tanner-Smith, E.E., 2015. Single-session alcohol interventions for heavy drinking college students: a systematic review and meta-analysis. J. Stud. Alcohol Drug 76, 530-543.

Saunders, J.B., Aasland, O.G., Babor, T.F., De La Fuente, J.R., Grant, M., 1993. Development of the Alcohol Use Disorders Identification Test (AUDIT): WHO Collaborative Project on early detection of persons with harmful alcohol consumption-II. Addiction 88, 791-804.

Schmajuk, M., Liotti, M., Busse, L., Woldorff, M.G., 2006. Electrophysiological activity underlying inhibitory control processes in normal adults. Neuropsychologia 44, 384-395.

Sheeran, P., Klein, W.M.P., Rothman, A.J., 2017. Health behavior change: moving from observation to intervention. Annu. Rev. Psychol. 68, null.
Smith, J.L., Mattick, R.P., Jamadar, S., Iredale, J.M., 2014. Deficits in behavioural inhibition in substance abuse and addiction: a meta-analysis. Drug Alcohol Depend. 145, 1-33.

Sobell, L.C., Sobell, M.B., 1992. Timeline follow-back: a technique for assessing self-reported alcohol consumption. In: Litten, R.Z., Allen, J. (Eds.), Measuring Alcohol Consumption: Psychosocial And Biological Methods. Humana Press, New Jersey, pp. 41-72.

van Deursen, D.S., Salemink, E., Smit, F., Kramer, J., Wiers, R.W., 2013. Web-based cognitive bias modification for problem drinkers: protocol of a randomised controlled trial with a 2x2x2 factorial design. BMC Public Health 13, 674.

Weafer, J., Fillmore, M.T., 2012. Alcohol-related stimuli reduce inhibitory control of behavior in drinkers. Psychopharmacology 222, 489-498.

Wiers, R.W., 2016. Cognitive bias modification does hold promise in the treatment of addiction: a commentary on Cristea, et al., 2016 PLoS One. PLoS One 11, e0162226 (comment). 